\title{
Generating Optimal Face Image in Face Recognition System
}

\author{
Yingchun Li, Guangda Su and Yan Shang \\ Electronic Engineering Department, Tsinghua University Beijing, \\ China
}

\section{Introduction}

Automatic face detection and recognition have been an active research area in the last two decades. There are urgent needs for face recognition in many practical applications, such as security monitoring, surveillance system and biometrics identified system. The majority of research has so far focused on frontal face and neutral expression face recognition. Recognizing faces reliably across changes in pose and expression has proved to be a much harder problem.

The most existing face recognition systems consist of single route image acquisition module with one camera. They follows the strategy of detection face images in complicated background, normalizing the detected face image and transmitting them to recognition module to be recognized. In real surveillance applications, because of the irregular head movement, most of the face images are of various pose and expression. The recognition rate is relatively low in such dynamic system. The evaluation of FRVT shows the level of performance for face verification of the best systems to be on par with face recognition for frontal faces. With increasing of the pose angle, the recognition rate decreases. The recognition rate decreases greatly when the pose angle is larger than 30 degree. This paper proposed a new method to improve the recognition rate by selecting and generating optimal face image from serial face images. Meanwhile, a new face recognition system with three route parallel modules is constructed. In order to get optimal face, it is necessary to estimate face pose and expression from the detected face images.

Pose estimation techniques can be classified into two main categories: model-based approaches and appearance-based approaches. The former use an explicit model of the face and recover the face pose based on the assumed model. A set of feature correspondences are established to estimate face pose. The latter directly use image pixels or features and assume that there exists a mapping relationship between face pose and certain properties of the facial image, which is constructed from a large number of training images. The popular eigenface approach is extended to handle multiple views and compared the performance of a parametric eigenspace with view-based eigenspace. Some researchers used model shape and texture nonlinearities across views in full 180 degree rotations. However, no face recognition experiments were performed. 3D can show face deep and topology information but at the cost of time. It is not suitable for real-time applications.

This paper is organized as follows. Section 2 introduces structure of the new face recognition system with optimal face image generation module. A new system module is built between

Source: Recent Advances in Face Recognition, Book edited by: Kresimir Delac, Mislav Grgic and Marian Stewart Bartlett, ISBN 978-953-7619-34-3, pp. 236, December 2008, I-Tech, Vienna, Austria 
face detecting module and face recognition module which is called optimal face generation module. The pose estimate method is explained in Section 3. Section 4 give a detailed explanation of the optimal face generation algorithm. Through combining the pose estimate method and adopting three routes parallel detecting system, we can select the optimal face image with little view rotation (when have appropriate view) or generate the face image with frontal view (when have no appropriate view). Experiments and conclusions are given in Section 5. The face recognition rate is improved after applying the proposed method and new system module.

\section{The system structure}

The proposed method is applied in parallel multi-route surveillance system. Its flow chart is shown in Fig.1. The system consists of four parts: multi-route face image acquisition module, multi-route face image detection module, optimal face generation module and face recognition module.

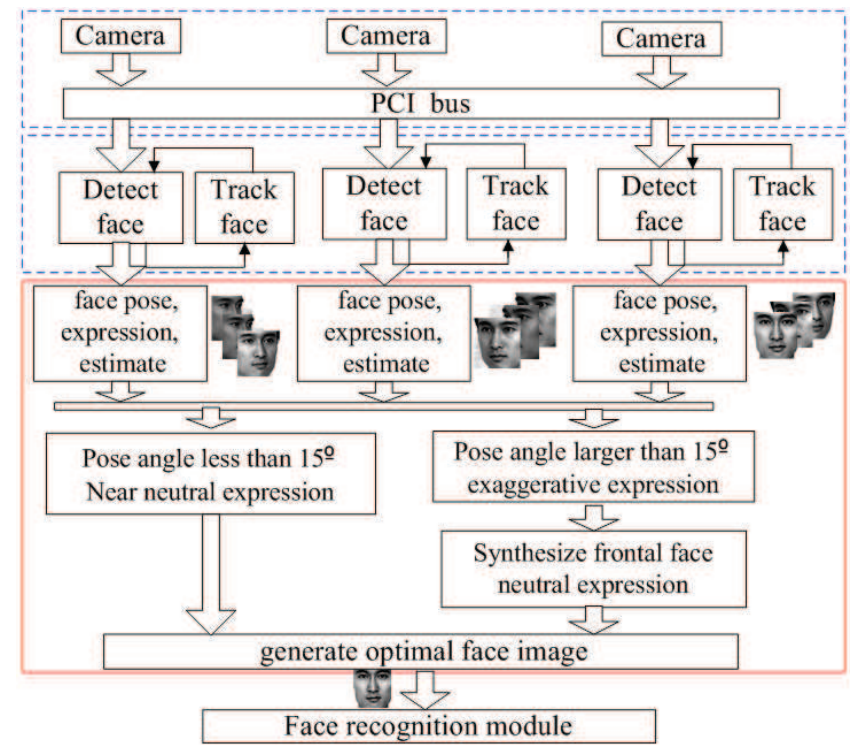

Fig. 1. Face recognition system.

The face image acquisition module consists of three cameras, image collective cards and PCI bus. It utilizes three cameras simultaneously capturing different pose angles from different face views in yaw. The image capturing device is shown in Fig. 2.

The three image streams are transmitted to the face detecting modules. Three cameras arranged in a row with equal intervals can extend detecting views and capture multiple face views in parallel. In Fig.2, we see that camera 1 can capture image with little pose angle even if the head is in a large rotation to camera 2. These performances are in need of real-time robust face detection module. The optimal face generation module is to select or synthesize best fit face to satisfy needs of recognition module by estimating the face pose and expression. FRVT shows that the recognition rate is high when pose angle is between $15^{\circ}$ and $-15^{\circ}$ (Set the frontal view is $0^{\circ}$ ) and it greatly decreases after the pose angle larger 
than $15^{\circ}$. Three cameras can enlarge rotation angles to $45^{\circ}$ towards right and left. This structure is able to solve the problem of face recognition with large pose angles.

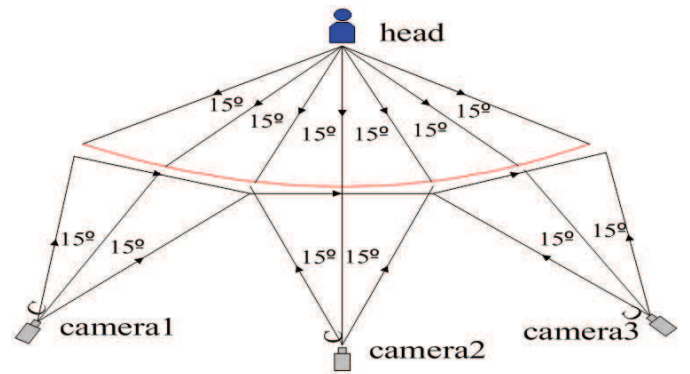

(a)

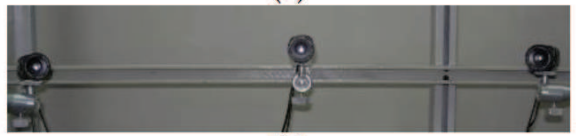

(b)

Fig. 2. Three-route face images captured device. (a)the configuration of cameras. (b) the image capturing device.

In the meantime, the optimal face generation module selects and synthesizes neutral facial expression from multiple detected face images. At last the optimal face image is acquired to be recognized in the following recognition module. This constructs a new face recognition system.

\section{The method of pose estimation}

In order to acquire optimal face with optimal face generation module, the first step is to estimate pose of the face images. The face images are preprocessed such as aligned, normalized, selected features. The system can automatically detect face locations of three feature points (two eyes and chin tip) and geometrically aligns the image to candidate poses training and test of the subjects. The examples of training images in TH (Tsinghua)database (built by ourselves) are shown in Fig.3.
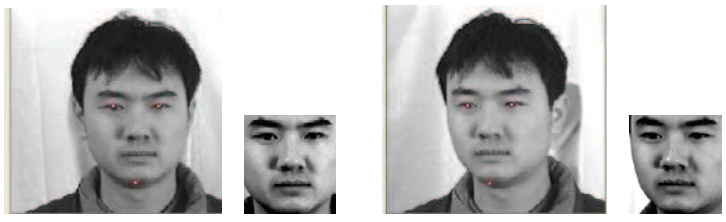

Fig. 3. The training face images in TH database.

It is essential to extract features from images utilizing the composite PCA (principle component analysis) and projecting face images to the eigenspace. Eigenvalue and eigenvector to each class can be calculated.

Given a set of samples $X_{i} \in \mathbb{R}^{N}$ represented face images by column vectors. The transformation matrix can be formed by using eigenvectors which normalized to unit matrix $T$.The projection of $X_{i}$ into the $\mathrm{N}$-dimensional subspace can be expressed as 


$$
\alpha=\left\{\alpha_{1}, \cdots, \alpha_{N}\right\}=X_{i}^{T} \cdot T
$$

SVM (support vector machine) is a learning algorithm for pattern classification. Its basic principle is to find the optimal linear hyperplane which the expected classification error for unseen test samples is minimized. According to the structural risk minimization principle, a function that classifies the training data accurately will generalize best regardless of the dimensionality of the input space.

Each training sample $x_{i}$ is associated with conefficient $\alpha_{i}$. Those samples whose coefficient $\alpha_{i}$ is nonzero are Support Vectors

Constructing an optimal hyperplane $W(\alpha)$ is to find all the nonzero $\alpha_{i}$. Any vector $x_{i}$ that corresponds to $\alpha_{i}$ is a support vector of the optimal hyperplane. $f(x)$ is an optimal classified function. $y_{i} \in(+1,-1)$.

$$
\begin{gathered}
f(x)=\operatorname{sgn}\left(\sum_{\text {vector }} y_{i} \alpha_{i}^{*} K\left(x_{i}, x\right)+b^{*}\right) \\
K\left(x_{i}, x_{j}\right)=\phi\left(x_{i}\right) \cdot \phi\left(x_{j}\right)
\end{gathered}
$$

Where the $K$ is a kernel function. Here we use linear kernel, $\phi\left(x_{i}\right)=x_{i}$, then $K\left(x_{i}, x_{j}\right)=x_{i} \cdot x_{j}=x_{i}^{T} x_{j}$.

Combining the above PCA and SVM classifier, we can draw better classification results. The samples were projected to eigenspace and the optimal hyperplane that correctly separates data points were found. Then the pose angle will be acquired for each face image.

The shape feature is shown in Fig.4. The feature points can give geometric characteristic. AB is the distance between two eyes when pose angle is $0, \mathrm{~A}^{\prime} \mathrm{E}$ is the distance between two eyes when pose angle is $\beta$. Set central angle $\alpha=2 \theta$, radius is unit 1 , so

$$
A^{\prime} E=2 \sin \theta \cos \beta=\sin (\theta+\beta)+\sin (\theta-\beta)
$$

Since distance $A B=2 \sin \theta$, then

$$
\beta=\arccos \left(\frac{\mathrm{A}^{\prime} \mathrm{E}}{\mathrm{AB}}\right)
$$

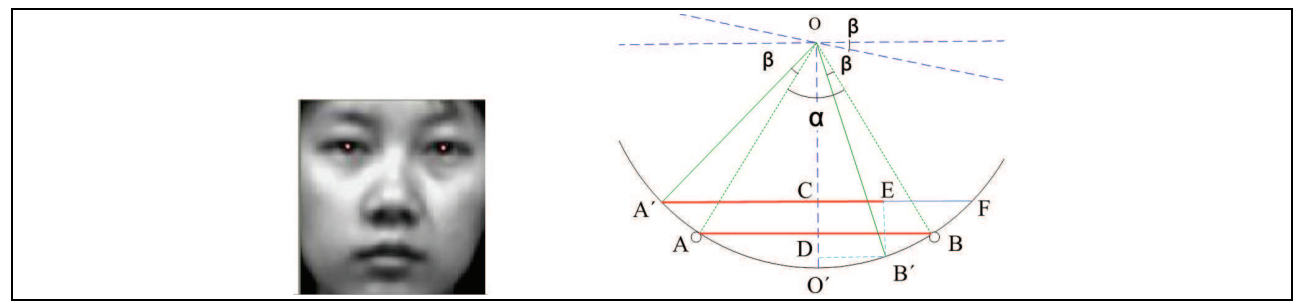

Fig. 4. Shape feature points and the configuration of pose variance.

We set weights of two vectors $\alpha$ and $\beta$ after two groups of features are gained. The combining PCA is expressed as: 


$$
\xi=p \cdot \alpha+q \cdot \beta \quad p+q=1
$$

Through selecting the suitable coefficient of weights, we get new eigenvector $\xi$.

Besides pose estimation, the human facial expressions are also estimated. After estimate the pose and expression, we can generate optimal face by multi-linear mappings over a set of vector spaces.

\section{Construction of optimal face generation}

Tensor is a multidimensional generalization of a matrix. A n-mode SVD (Singular Value Decomposition) can decompose an n-dimensional tensor $\mathbb{F}$ into the n-mode product of $\mathrm{N}$ orthogonal spaces. The notion of tensor can be applied to a face image in the following way. Consider a set of $U_{\text {pixels }}$ images of $U_{\text {peoples }}$ people's faces, each image in $U_{p}$ poses, with $U_{e}$ expressions. The facial image tensor can be decomposed into

$$
\mathbb{F}=\mathbb{Z} \times{ }_{1} U_{\text {pixels }} \times_{2} U_{\text {peoples }} \times{ }_{3} U_{p} \times{ }_{4} U_{e}
$$

Each mode matrix represents a parameter. The columns of $U_{p}$ and $U_{\mathrm{e}}$ respectively span the space of pose and expression parameters. The columns of $U_{\text {pixels }}$ span the image space. These are the eigenfaces obtained by PCA on the whole data set. The core tensor $\mathbb{Z}$ governs the interactions between these mode matrices and represents only the principal axes of variation over all images.

Each person can be represented by the same coefficient vector regardless of pose and expression. It is expressed as

$$
\begin{gathered}
\mathbb{F}=\mathbb{S} \times{ }_{2} U_{\text {peoples }} \\
\mathbb{S}=\mathbb{Z} \times{ }_{1} U_{\text {pixels }} \times_{3} U_{p} \times_{4} U_{e}
\end{gathered}
$$

$\mathbb{S}$ defines different bases for each combination of pose and expression and $U_{\text {peoples }}$ contains the coefficients. Tensor $\mathbb{S}$ can be indexed into a particular pose and expression to obtain a subtensor $\mathbb{S}_{p, e}$. Then the subtensor $\mathbb{S}_{p, e(\text { peoples })}$ was flattened along identity (people) mode, which column vectors span the space of images under particular pose and expression. Subtensor $\mathbb{S}_{p}, \mathbb{S}_{e}$ are in the maximum projection direction of which $\mathbb{S}_{p, e}$ projects in various types of eigenspace. Given an input image, the estimate value is computed by projecting into correlative subtensor such as pose, expression, and then identify them relatively.

The frontal faces or the faces which pose angle is less than $15^{\circ}$ (radian is $\pi / 12$ ) are the optimal face with optimal pose. If the pose angle of face image is over $15^{\circ}$, the frontal view can be synthesized by several multi-view face images in morphable model method. The optimal pose view $U_{o p_{-} p}$ is expressed as

$$
U_{o p_{-} p}=\frac{1}{2}\left\{U_{p}\left[\operatorname{sgn}\left(\frac{\pi}{12}-\left|U_{p}\right|\right)+1\right]+U_{p s}\left[\operatorname{sgn}\left(\left|U_{p}\right|-\frac{\pi}{12}\right)+1\right]\right\}
$$

Where sgn is sign function, $U_{p}$ is pose parameter, $U_{p s}$ is synthesis parameter. 
The same idea is also applied in expression estimate. Neutral expressions or little variation expressions are the optimal expression. So the faces with exaggerated expression can be synthesized to normal expression to generate optimal expression $U_{o p_{-} e}$.

The optimal face image is the well-posed (the yaw pose angle is within $15^{\circ}$ ) image with neutral facial expression or little variation expression. The optimal face $B_{o p_{-} \text {face }}$ is expressed as

$$
B_{o p_{-} \text {face }}=\mathbb{Z} \times{ }_{1} U_{\text {peoples }} \times_{3} U_{o p_{-} p} \times_{4} U_{o p_{-} e}
$$

The optimal face image can reduce high dimensions which caused by the effects of uncertain factors such as pose and expression. It can simplify the design of the classifiers and improve their performance.

\section{Experimental results}

We used three databases in our system across pose experiments, still face images of TH database, CMU PIE database and video images of TH database. Fig.5 and Fig.6 show parts of results of pose estimation of still images in CMU PIE database and video images in TH database. The comparison results of the three tests are in Table 1.

\begin{tabular}{|c|c|c|c|}
\hline Tatabase & images & Correct & Correct rate \\
\hline TH database & 1080 & 1059 & $98.1 \%$ \\
\hline CMU_PIE & 400 & 386 & $96.9 \%$ \\
\hline TH video & 395 & 379 & $95.9 \%$ \\
\hline
\end{tabular}

Table 1. Comparison of pose estimation in three databases.

Adopting the method of generating optimal face image can acquire a best fit face image among serial images. The recognition rate of still images is higher than that of video images. Because the training images are original from $\mathrm{TH}$ database, the quality of still image is better than that of video images. The recognition rate of still images in TH database is higher than that in CMU PIE database. We compare another performance of the proposed system. The test is between three-route parallel face recognition system with optimal face image generation module and single-route face recognition system. The test results are shown in Table 2.

\begin{tabular}{|c|c|c|}
\hline Recognition rate & Single route & Three route \\
\hline TH Standard & $82.57 \%$ & $85.71 \%$ \\
\hline FRVT Standard & $78.29 \%$ & $80.80 \%$ \\
\hline
\end{tabular}

Table 2. Comparison of recognition rates in face system.

We test recognition rates in dynamic face system according to TH standard and FRVT standard respectively. We have tested 656 individuals in pose angle no more than $60^{\circ}$ (towards left and right). The experiment results show that three-route parallel system gets higher recognition rate and outgoes single system no matter which standard is adopted. FRVT reported the test in database of 87 individuals which only have pose variance. When the pose view reached $30^{\circ}$ or $45^{\circ}$, its recognition rate decreased to $45 \%$. But our proposed 
method overcomes the problem which large pose angles affect face recognition rate. It helps to increase recognition rate and improve performance of system.
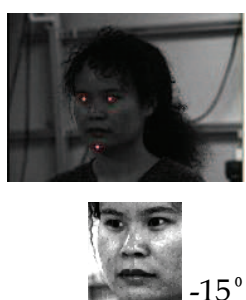
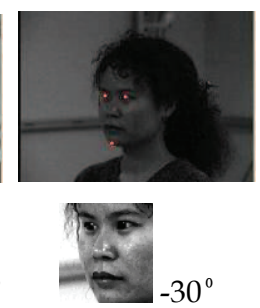
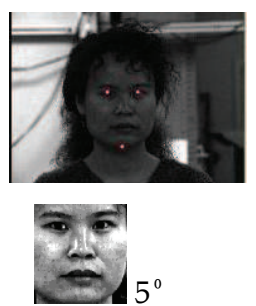

Fig. 5. Pose estimation results in CMU PIE database.
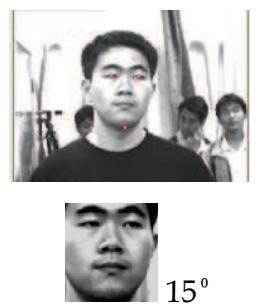
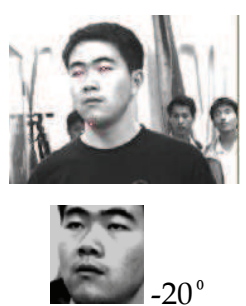
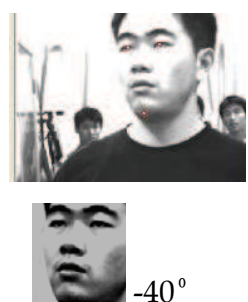

Fig. 6. Pose estimation results in surveillance system.

The key point of face recognition is to acquire optimal face image. The aim of generating optimal face image is to form a fit image for recognition which has minimum pose angle and neutral expression in a serial dynamic face images.

In fact, the optimal face image generation module is a new integrated preprocessing module in face recognition system. Its idea can extend to other factors such as illumination and resolution and so on. The experiment results show that proposed method is feasible and effective. The optimal face image generation module is indispensable in the face recognition system. Future research will focus on improving its calculating speed to meet the needs of the real-time application.

\section{Conclusion}

The theory about discrimination of $2 \mathrm{D}$ optimal face is to improve recognition rate in dynamic system. In order to overcome the effects of face pose, expression, illumination and resolution, it is essential to select a $2 \mathrm{D}$ optimal face and transmit it to the dynamic face recognition system to be recognized. The discriminate system of $2 \mathrm{D}$ optimal face is built between face detecting and face recognition system. It depends on the facial pose estimate, expression estimate, lighting estimate and the computation of detected facial area.

\section{References}

Phillips, P.J., Grother, P., Micheal, R., Blackburn, D.M., Tabassi, E. \& Bone, M., (2003). Face Recognition Vendor Test 2002, Analysis and Modeling of Faces and Gestures, Arlington, USA. ISBN: 0-7695-2010-3. 
Gao, Y., Leung, M. \& Wang, W., (2001). Fast face identification under varying pose from a single 2-D model view, IEE Proceedings of Vision Image Signal Process. Vol. 148, No.4.

Ji, Q., (2002). 3D Face pose estimation and tracking from a camera, Image and Vision Computing, Vol. 20, No.7, pp.499-511.

Turk, M., Pentland, A., (1991). Face Recognition Using Eignefaces, IEEE Conference on Computer Vision and Pattern Recognition, pp. 586-591.

$\mathrm{Gu}, \mathrm{H} ., \mathrm{Su}, \mathrm{G}$. \& Du, C. (2003). Feature Points Extraction from Faces, Image and Vision Computing, pp.154-158.

Vapnik, V., (1998) .Statistical Learning Theory, John Wiley, New York. ISSN: 0-471-03003-1

Sim, T.,Baker, S. \& Bsat, M., (2003). The CMU Pose, Illumination, and Expression Database, IEEE Transactions on Pattern Analysis and Machine Intelligence, Vol. 25, No.12, pp.1615-1618. ISSN: 0162-8828. 


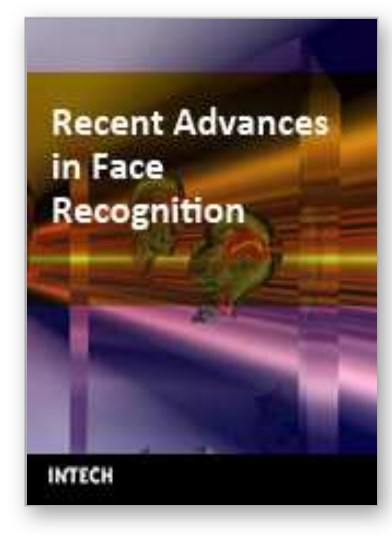

\section{Recent Advances in Face Recognition \\ Edited by Kresimir Delac, Mislav Grgic and Marian Stewart Bartlett}

ISBN 978-953-7619-34-3

Hard cover, 236 pages

Publisher InTech

Published online 01, June, 2008

Published in print edition June, 2008

The main idea and the driver of further research in the area of face recognition are security applications and human-computer interaction. Face recognition represents an intuitive and non-intrusive method of recognizing people and this is why it became one of three identification methods used in e-passports and a biometric of choice for many other security applications. This goal of this book is to provide the reader with the most up to date research performed in automatic face recognition. The chapters presented use innovative approaches to deal with a wide variety of unsolved issues.

\section{How to reference}

In order to correctly reference this scholarly work, feel free to copy and paste the following:

Yingchun Li, Guangda Su and Yan Shang (2008). Generating Optimal Face Image in Face Recognition System, Recent Advances in Face Recognition, Kresimir Delac, Mislav Grgic and Marian Stewart Bartlett (Ed.), ISBN: 978-953-7619-34-3, InTech, Available from:

http://www.intechopen.com/books/recent_advances_in_face_recognition/generating_optimal_face_image_in_f ace_recognition_system

\section{INTECH}

open science | open minds

\section{InTech Europe}

University Campus STeP Ri

Slavka Krautzeka 83/A

51000 Rijeka, Croatia

Phone: +385 (51) 770447

Fax: +385 (51) 686166

www.intechopen.com

\section{InTech China}

Unit 405, Office Block, Hotel Equatorial Shanghai

No.65, Yan An Road (West), Shanghai, 200040, China

中国上海市延安西路65号上海国际贵都大饭店办公楼 405 单元

Phone: +86-21-62489820

Fax: $+86-21-62489821$ 
(C) 2008 The Author(s). Licensee IntechOpen. This chapter is distributed under the terms of the Creative Commons Attribution-NonCommercialShareAlike-3.0 License, which permits use, distribution and reproduction for non-commercial purposes, provided the original is properly cited and derivative works building on this content are distributed under the same license. 